\title{
Processing level and recognition: A search method for inducing alternative encodings of unrelated words
}

\author{
FRANK J. DaPOLITO \\ University of Dayton, Dayton, Ohio 45409 \\ and \\ CHRIS HALE and ROBIN L. SHEW \\ Systems Research Laboratories, Inc., Dayton, Ohio 45440
}

\begin{abstract}
Twenty-four subjects viewed cards containing four words. In synonym orienting, half of the cards contained a synonym pair and half did not; in homophone orienting, half of the displays contained a homophone pair. The remaining two words in each display were used to index retention. During presentation of displays, subjects responded "yes" or "no" to indicate the presence or absence of a matching pair. Following presentation, a recognition test was administered. Half the items were tested early and half were tested late; within each test order, half the items appeared with context cues during testing and half appeared without. Results showed superior recognition (1) following semantic orienting, (2) for items tested early, (3) for items tested with context, and (4) for nonmatch displays requiring exhaustive item comparison. An Orienting by Test Cuing interaction showed that the presence of test cues improved recognition for homophone orienting items only. The interaction may have resulted from the lack of cuetarget associations and the reinstatement of the phonemic orienting set during the test.
\end{abstract}

Contemporary studies of memory have attempted to gain some measure of direct control over the encoding process used by subjects during acquisition. One method that is often used requires the subject to perform some orienting task during item presentation. This method assumes that different attributes of the nominal stimuli will be selectively attended to and processed, depending upon whether the orienting task requires orthographic, phonemic, or semantic discriminations. According to Postman (1976), orienting tasks that are carried out under conditions of incidental learning (i.e., no explicit instructions to learn are given) are especially potent in determining the subject's encoding process.

Evidence that the type of orienting task does determine subsequent memory performance has been provided by a number of investigators (Craik \& Tulving, 1975; Hyde \& Jenkins, 1969; Walsh \& Jenkins, 1973). The studies by Jenkins and his colleagues were among the first to establish that tasks involving "semantic judgments" produced recall performance that was equal to that of intentional learners and superior to tasks involving "nonsemantic" judgments. The result has been replicated many times (Hyde, 1973; Jenkins, 1974; Till, Diehl, \& Jenkins, 1975).

By far, the dominant interpretation of experiments showing an orienting effect has been in terms of the "levels-of-processing" framework (Craik \& Lockhart, 1972). According to this view, encoding processes are hierarchically organized so that verbal stimuli are initially coded in terms of orthographic structure (shallow encoding), analyzed with respect to phonemic and syllabic features, subsequently analyzed with respect to word meanings, and, finally, if the learning materials permit, elaborated or coded into higher order semantic structures (deep encoding) involving relations among concepts. The integrity and durability of the memory trace for a given item is presumed to be directly related to the level of processing that item has received. Admittedly, mixed codes (e.g., orthographic, phonemic, and semantic) might be generated for a given input word, but it is assumed that an effective orienting task enables one type of encoding process to predominate during presentation.

Postman (1976) has discussed some problems of methodology in the early studies employing orienting tasks. First, he questioned the validity of an intuitive classification of various orienting tasks. For example, Postman (1976, p. 37) states that "it is not self evident that one has to engage in extensive semantic processing in order to judge the frequency of a word, nor is it obvious that one can entirely ignore meaning when making decisions about parts of speech." Moreover, he suggests that the effects of orienting instructions are often confounded with other important variables that influence recall and recognition. For example, many so-called semantic tasks require a subjective rating of the critical words along a broad dimension (e.g., pleasantness), and this might induce subjects to "look 
back" through the presentation words in order to make comparisons among items. Any such "displaced rehearsal" due to subjective interitem comparisons would greatly improve retention. On the other hand, many nonsemantic tasks involve judgments that can be made by viewing an individual input word in isolation or even a portion of a word (e.g., vowel or case judgments). Consequently, different amounts of rehearsal may be confounded with the specific type of coding presumed to be fostered by the orienting instructions.

A further issue raised by Postman (1976) is that, in some of the earlier research, the semantic orienting tasks required subjects to make decisions about key items that are not objectively "right" or "wrong" (e.g., pleasantness), whereas the nonsemantic task (vowel, rhyme, or case discrimination) could be objectively correct or incorrect. This difference among orienting tasks may have required different "amounts of information reduction" or attention capacity across the various tasks. Consequently, semantic and nonsemantic tasks may have differed in difficulty and capacity for producing interference.

Craik and Tulving (1975) developed a method for producing "item-specific" encoding that attempted to avoid some of the difficulties mentioned by Postman (1976). Prior to each word presentation, they asked a question about the word that could be objectively answered by interpreting the item along an orthographic, phonemic, or semantic dimension (e.g., "___ rhymes with dog:" followed by the target word "log"). Their results generally agreed with the levels-of-processing position, except for the fact that the same orienting task produced different retention of items for "yes" and "no" questions. Craik and Tulving (1975) suggest that for "yes" questions, the target words are more related to the question and this "compatibility effect" (Schulman, 1971) produces a more integrated retrieval unit. They also suggest that at the semantic level of orienting, there could be different degrees of elaboration of the semantic code and that degree of elaboration may be a more important determinant of retention than is depth of encoding. At least for semantic orienting, the degree and speed of elaboration may be much greater for words presented with meaningful "yes" questions (e.g., "Say yes or no. A ___ has feathers:" followed by the target word "eagle"). However, if the question "A ___ has feathers?" is followed by the target word "boy" or "train," then the correct answer is "no" but the sentence becomes semantically anomalous or even "meaningless." Although individual words are never "meaningless," sentences may or may not be, and this may influence the degree of elaboration and retrievability of the critical items embedded in "yes" or "no" questions.

Recently, Postman, Thompkins, and Gray (1978) questioned the interpretation of experiments that use the "encoding question" method developed by Craik and Tulving (1975). According to Postman et al. (1978), the orienting questions may prime or activate preexperimental associations that exist between the target word and some of the sentence words, and the amount of priming may differ across orienting tasks. For example, in the semantic orienting question presented above, a strong association between the words "feathers" and "eagle" already exists, and this association may be stronger than that between the words "dog" and "log" in the phonemic orienting question presented above. In effect, the superiority of semantic over phonemic orienting obtained in many studies may have been inflated or predetermined by confounding orienting task with the transfer effects of prior learning. Postman et al. (1978) suggest that these potential biases may be avoided by (1) using orienting tasks that eliminate preexperimental associations between encoding items and critical items and (2) holding the learning materials constant across different orienting tasks.

Although it may prove to be impossible to manipulate encoding under incidental learning conditions without influencing other important retention and retrieval variables, the present experiment attempts to remove at least some of the difficulties described by Postman (1976). An attempt was made to insure phonemic or semantic encoding of individual words without the complications involved with sentence embedding tasks, such as question-target compatibility or question uniqueness and the like.

This study used a modified "search" task in which displays containing four words were briefly presented. In each display, subjects attempted to find two synonyms (e.g., urn-vase) among the four words or two homophones (urn-earn) in the display of four items. One half of the displays actually contained a match, and the remaining displays did not. The synonym or homophone pairs contained in the positive-match displays and their equivalents in the nonmatch displays were of no particular interest; rather, the two remaining context words were used to index retention under the homophonic or semantic search tasks. Presentation lists were balanced so that the same critical target words occurred under the semantic and phonemic orienting conditions. Consequently, the orienting tasks induced the processing of the same critical words in one of two ways in order for the subject to make a correct decision during display presentation. Although this search task does not preclude the subject's encoding other dimensions of the items, it does insure that a correct yes-no response must be based upon the outcome of word comparisons along either a semantic or phonemic dimension. Also, for some of the positive-match displays, subjects could respond correctly without necessarily making comparisons among all four words, but a correct response to a nonmatch display required exhaustive comparison. Thus, for a given type of encoding task, different degrees of "looking back" might be produced, and the retention effects of this differential rehearsal may be evaluated for each orienting task. During tests, critical words were tested 
either with or without the synonyms (homophones) present on the test in order to evaluate the effects of retrieval cues for items acquired under semantic vs. phonemic orienting. Finally, critical items were tested either in the first half or in the last half of the test phase. This manipulation was an attempt to determine whether identical words acquired under the two orienting tasks might show different rates of decay over the test phase.

\section{METHOD}

\section{Subjects}

Twenty-four introductory psychology students at the University of Dayton served as subjects. Their participation in experiments satisfied a course requirement.

\section{Design}

The experiment was a 2 by 2 by 2 by 2 mixed design with the first factor, orienting task, varied between subjects and the remaining three factors, test interval (early vs. late), condition of testing (cues vs. no cues), and input display type (positive match vs. nonmatch), varied within subjects.

\section{Apparatus and Materials}

A two-channel tachistoscope (Scientific Prototype Model $800 \mathrm{~F}$ ) was used to present the display cards. Reaction time to each display was recorded to the nearest $1 \mathrm{msec}$ with a voice key that triggered a Hunter timer (Model 120 C).

The stimuli were two decks of $245 \times 8$ in. white cards, each card containing four English words. Each input display contained two "critical words" taken from the Thorndike-Lorge (1944) word frequency count (occurrence from 30 to AA) and two "orienting task words." The orienting words were either synonyms or homophones of each other or two unrelated words that each had a homophone and synonym not present in the display.

The input displays were developed by first selecting a basic pool of 100 homophone pairs taken from Whitford (1966). This selection produced approximately 35 homophones that started with different first letters (scent, cent) and 65 that started with the same first letter (berth, birth). Then, for each word of a homophone pair, the dictionary was used to find a single-word synonym or "close" synonym (e.g., smell, penny, bunk, origin). Two independent sets of 24 quadruplets were constructed from this basic pool, as described below.

Input List $1 \mathrm{~A}$ for homophone orienting was constructed by assigning 12 of the 35 homophone pairs starting with different letters to the positive-match displays. Since homophones are orthographically similar, we used words with different first letters so that the subjects in homophone orienting could not employ the strategy of first-letter cuing. Two critical highfrequency words were then assigned to each of the 12 homophone displays, such that a typical positive-match item would be "scent, child, cent, glass." For each of the 12 nonmatch displays, two critical high-frequency words and two different homophones were assigned to form a corresponding input display (e.g., sew, cake, fowl, bell). The four words were then typed in a column so that each of the six possible positions of the two orienting words was represented twice in each group of 12 displays. Input List $1 \mathrm{~B}$ for synonym orienting was formed by substituting a synonym for one of the homophones in List $1 \mathrm{~A}$ (e.g., penny, child, cent, glass) and keeping the same nonmatch display. Thus, the positive-match displays in Lists $1 \mathrm{~A}$ and $1 \mathrm{~B}$ differed by one noncritical orienting word, whereas the nonmatch displays were identical.

Using the same principles, a second set of materials (Lists $2 \mathrm{~A}$ and $2 B$ ) was constructed with a completely different set of words. Thus, each List $2 \mathrm{~A}$ and $2 \mathrm{~B}$ contained 24 displays (12 match and 12 nonmatch), with each display containing two critical items and two "orienting" items. For each display, the four words were typed in a column with each of six permutations of orienting word positions represented twice. The test materials were four mimeographed sheets, each containing 12 multiple-choice items.

Each of the 48 four-choice test items contained one critical word and three high-frequency distractor words, with the position of the critical word randomly determined. Finally, one-half of the choice items on each sheet were cued by having the original input display present, with the critical word positions replaced by dashes. The context cue, if present, was vertically typed to the left of the row. For example, "scent, , cent, __ " would be a test cue for a homophone orienting positive-match item, whereas "sew, ___, fowl, "would be a test cue for a homophone (also synonym) nonmatch display item.

\section{Procedure}

Subjects were tested individually, and each received a single presentation trial followed by a test trial. Each presentation card was presented for $1 \mathrm{sec}$, with an intercard interval of approximately 5 sec.

All subjects were informed that they were in a language skill experiment designed to test how quickly and accurately they could read word groups in order to find words that were alike in sound (homophone orienting group) or meaning (semantic orienting group). The subjects were told that half of the displays would contain words that were alike in sound (or meaning) and half would not. They were instructed to respond "yes" if there was a matching pair of items in the display and "no" otherwise. Each presentation was preceded by a verbal "ready" signal, and reaction times were recorded for each display.

After all 24 displays were presented, subjects were given a mimeographed test booklet containing 48 multiple-choice items. Subjects were told that each row of four words contained one word that had been presented in one of the input displays. Subjects were to view each row and then encircle the word that they believed had been presented. Subjects were told that half of the test items contained a column of two words and two dashed lines to the left margin and half of the test rows did not. They were to study these cues if present, since the cue was a part of the original input item and this might help them select a correct test word. Subjects worked through the booklet at their own pace.

\section{RESULTS}

\section{Orienting Decision Latency}

Since there were only three incorrect orienting decisions in the total experiment, all response times were used in the following analysis: For each subject in each orienting condition, a mean decision latency was obtained separately for the 12 match and 12 nonmatch input displays. For the semantic orienting group, these latencies were $2.32 \mathrm{sec}$ and $2.62 \mathrm{sec}$ for match displays and nonmatch displays, respectively. The decision latencies in the homophone condition were $1.99 \mathrm{sec}$ for match items and $2.24 \mathrm{sec}$ for nonmatch items. Thus, decision times for synonyms required about $1 / 3 \mathrm{sec}$ longer than those based on phonemic comparisons. Similarly, for each orienting condition, nonmatch response times were longer than those for match displays. Analysis of variance confirmed the above description. 
The main effect of orienting task was significant $[F(1,22)=25.84, p<.001]$, and the difference between display types was also significant $[F(1,22)=15.45$, $p<.001]$. There was no interaction between these variables.

In general, the decision times during presentation are directly correlated with the overall accuracy of subsequent recognition. However, it has been argued in the past that neither time nor effort can necessarily account for the superior effects of semantic orienting (Craik \& Tulving, 1975; Hyde, 1973; Walsh \& Jenkins, 1973). Consequently, a discussion of decision times will not be attempted.

\section{Recognition of Nonmatch Display Items}

The critical data relative to the levels-of-processing point of view come from an analysis of the nonmatch items. Postman et al. (1978) suggested that it might be possible to assess level of processing per se by (1) eliminating known preexperimental associations between orienting items and critical target items in order to avoid priming and transfer effects, (2) holding the critical materials constant across encoding tasks, and (3) employing orienting tasks that objectively show that subjects correctly performed the task at the appropriate "level of processing." Only the nonmatch displays yield data under conditions in which identical displays were presented under semantic and phonemic orienting instructions; the same unrelated orienting words were used as test cues, and the same critical words were tested for incidental recognition.

The proportions of nonmatch items recognized are summarized in Table 1. Entries are presented for each combination of orienting task, test cuing condition, and order to testing. Assuming that subjects without knowledge guess randomly among the four alternatives, chance performance would be .25 .

Inspection of Table 1 shows the clear superiority of semantic over homophone orienting (.70 vs. .48). Also, items tested in the first half of the test block were superior to items tested later, and the presence of context cues during the test improved recognition performance for only the homophone orienting condition.

An analysis of variance on recognition scores for nonmatch displays showed significant effects for orienting task $[F(1,22)=28.79, p<.001]$, test interval

Table 1

Proportions of Correct Recognitions for Nonmatch Display Items

\begin{tabular}{cccccc}
\hline & \multicolumn{4}{c}{ Test } \\
\cline { 2 - 3 } $\begin{array}{c}\text { Orienting } \\
\text { Condition }\end{array}$ & \multicolumn{2}{c}{ Context Cues } & & \multicolumn{2}{c}{ No Context Cues } \\
\cline { 5 - 6 } & Early & Late & & Early & Late \\
\hline Synonym & .75 & .65 & & .76 & .65 \\
Homophone & .61 & .57 & & .43 & .32 \\
\hline
\end{tabular}

Table 2

Proportions of Correct Recognitions for Match Display Items

\begin{tabular}{lccccc}
\hline & \multicolumn{4}{c}{ Test } \\
\cline { 2 - 3 } $\begin{array}{l}\text { Orienting } \\
\text { Condition }\end{array}$ & \multicolumn{2}{c}{ Context Cues } & & No Context Cues \\
\cline { 5 - 6 } & Early & Late & & Early & Late \\
\hline Synonym & .63 & .68 & & 63 & .46 \\
Homophone & .36 & .33 & & .39 & .19 \\
\hline
\end{tabular}

$[F(1,22)=7.25, p<.01]$, and test cuing condition $[\mathrm{F}(1,22)=5.45, \mathrm{p}<.03]$. Also, the Orienting Task by Test Cuing interaction was significant $[F(1,22)=6.18$, $p<.02]$. No other interactions were obtained.

\section{Recognition of Match vs. Nonmatch Items}

The proportions of correct recognitions for words embedded in match displays are presented in Table 2. A comparison of these entries with those of Table 1 shows that overall recognition was lower for the match displays that did not necessarily require reading all four words during orienting. Unfortunately, there was a floor effect (essentially chance performance) for the homophone orienting items that were tested late and without cues. However, there was again a clear superiority of semantic over phonemic orienting.

A four-way mixed analysis of variance was performed on the data from Tables 1 and 2 combined. Thus, orienting task varied between subjects, and display type (match vs. nonmatch), test interval (early vs. late), and condition of testing (cues vs. no cues) were withinsubjects factors. This analysis showed that all main effects were significant.

The results were as follows: for orienting task, $\mathrm{F}(1,22)=56.99(\mathrm{p}<.001)$; for display type, $\mathrm{F}(1,22)=$ $26.02(p<.001)$; for test interval (early vs. late), $F(1,22)=10.59(p<.01)$; and for test cuing condition, $F(1,22)=9.31 \quad(p<.004)$. Surprisingly, there were no significant two-way interactions, although the Test Cuing by Test Interval interaction was marginal $(\mathrm{p}<.08)$. The single significant interaction was the three-way interaction involving orienting task, test cuing condition, and input display type. This result appears to be due to an Orienting by Test Cuing interaction that occurred for nonmatch displays only.

\section{DISCUSSION}

In this study, depth of processing was operationally defined in terms of two search tasks. Each task required comparisons among four words in order to generate a yes-no decision based upon the presence or absence of either a synonym or a homophone match. For each orienting condition, the nonmatch displays contained identical items, guaranteed that all words were compared during presentation, contained no preexperimental associations among any of the words, and produced 
recognition performance that was above chance in all conditions. Thus, the most appropriate data came from the nonmatch display items, and the discussion will concentrate upon these data.

First, the results exhibit the typical superiority of semantic over nonsemantic processing, and it is apparent that this "levels" effect cannot be easily attributed to differences in acquisition materials, congruity, rehearsal processes, or the priming of preexperimental associations. Insofar as the particular conditions of testing used in this study are valid, these data lend strong support to the levels-of-processing approach.

However, it is possible that the recognition tests employed were not appropriate measures and that a clear-cut assessment of performance following different levels of processing is going to be a very difficult problem. For example, Morris, Bransford, and Franks (1977) have argued that conventional tests of recall and recognition may not necessarily give an unbiased evaluation of the kinds (and amounts) of information acquired under different orienting conditions. Using the "encoding question" method, Morris et al. (1977) showed that semantic acquisition was superior to rhyme acquisition when subjects were tested by a standard recognition test, whereas rhyme orienting was superior to semantic orienting when a recognition test for rhymes was given. It is clear that a replication of the Morris et al. (1977) design with our procedure might be instructive, since our nonmatch displays contain unrelated words and also permit one to test for a homophone of an orienting word, a synonym of an orienting word, and an original target word as well. Although it may be difficult to agree upon an operational definition of "test appropriateness," Morris et al. (1977) point to a potentially serious problem. It may well be the case that some conventional methods of testing (ours included) are inappropriate to the orienting task.

The second major result of this study clearly appears to be associated with processes occurring during the test. In particular, the interaction of orienting task with test cuing indicated that the presence of test cues identical to those of acquisition improved recognition for homophone orienting but had no influence whatever for synonym orienting items. Taken at face value, this result is inconsistent with the levels-of-processing view. For example, Fisher and Craik (1977) claim that the presence of the original training cues during the test (an optimal retrieval environment) should have a greater retrieval effect when the encoding is "deep" or "elaborative" (i.e., semantic), and their data support this. Apparently, our result suggests that with unrelated items and minimal acquisition, the reinstatement of phonemic cues aids retrieval more than the reinstatement of semantic information does. Thus, context cuing attenuated the levels-of-processing effect. Unfortunately, this interaction occurred only with the nonmatch displays, and it could be a chance result. However, the interaction occurred strongly in both sets of materials (List 1 and List 2), and we will tentatively assume that it is a real effect.

The interaction appears to be due to two factors: the use of unrelated items during encoding and the relative degree of test appropriateness for the two orienting conditions. For subjects engaged in semantic orienting, the uncued recognition test was quite appropriate in the sense that strong predispositions or habits to encode words semantically were carried over from orienting to the test. The addition of test cues had no effect for semantic orienting because there were no effective cue-target associations established at input or before and, in addition, the cues did not act to further reinstate an appropriate encoding set that was already present. In contrast, subjects in the homophone orienting condition did not have the benefit of the transfer of a phonemic set from orienting to the test. In fact, the uncued recognition items may have disposed homophone subjects to drift back into a semantic retrieval mode, resulting in performance that was substantially below that in the uncued semantic condition. In short, the levels-of-processing effect for uncued items may have been substantially inflated. However, providing the subjects with retrieval cues that often could be easily recognized as having homophones (e.g., kernel, dew, ) reinstated the appropriate encoding set and improved recognition. Postman et al. (1978) have raised the possibility that strong dispositions or habits to code meaning are primed during conventional recognition tests. Clearly, if conventional recognition tests always prime subjects to process familiar words semantically, then part of the superiority of semantic processing is an artifact due to a mismatch between encoding processes during presentation and those at the test. Again, more research along the lines of Morris et al. (1977) is needed to obtain a clearer answer on this point. The problem of maintaining the same encoding set from acquisition to the test phase might be an interesting subject for future research.

\section{REFERENCES}

CRAIK, F. I. M., \& LockharT, R. S. Levels of processing: A framework for memory research. Journal of Verbal Learning and Verbal Behavior, 1972, 11, 671-684.

Craik, F. I. M., \& Tulving, E. Depth of processing and the retention of words in episodic memory. Journal of Experimental Psychology: General, 1975, 104, 268-294.

Fisher, R. P., \& Craik, F. I. M. Interaction between encoding and retrieval operations in cued recall. Journal of Experimental Psychology: Human Learning and Memory, 1977, 3, 701-711.

HyDE, T. S. Differential effects of effort and type of orienting task on recall and organization of highly associated words. Journal of Experimental Psychology, 1973, 97, 111-113.

Hyde, T. S., \& Jenkins, J. J. Differential effects of incidental tasks on the organization of recall of a list of highly associated words. Journal of Experimental Psychology, 1969, 82, 472-481.

Jenkins, J. J. Can we have a meaningful theory of memory? In R. L. Solso (Ed.), Theories in cognitive psychology: The Loyola symposium. Hillsdale, N.J: Erlbaum, 1974.

Morris, C. D., Bransford, J. D., \& Franks, J. J. Levels of 
processing versus transfel appropriate processing. Journal of Verbal Learning and Verbal Behavior, 1977, 16, 519-533.

Postman, L. Methodology of human learning. In W. K. Estes (Ed.), Handbook of learning and cognitive processes (Vol. 3). Hillsdale, N.J: Erlbaum, 1976.

Postman, L., Thompkins, B. A., \& Gray, W. D. The interpretation of encoding effects in retention. Journal of Verbal Learning and Verbal Behavior, 1978, 17, 681-705.

Schulman, A. I. Recognition memory for targets from a scanned list. British Journal of Psychology, 1971, 62, 335-346.

ThORNDIKE, E. L., \& LORGE, I. The teacher's word book of 30,000 words. New York: Columbia University, Teachers College, Bureau of Publications, 1944.
Till, R. E., Diehl, R. L., \& Jenkins, J. J. Effects of semantic and nonsemantic cued orienting tasks on associative clustering in free recall. Memory \& Cognition, 1975, 3, 19-23.

WALSh, D. A., \& JENKins, J. J. Effects of orienting tasks on free recall in incidental learning: "Difficulty," "effort," and "process" explanations. Journal of Verbal Learning and Verbal Behavior, 1973, 12, 481-488.

Whitrond, H. C. A dictionary of American homophones and homographs. New York: Teachers College Press, 1966.

(Received for publication May 9, 1980; revision accepted September 4, 1980.) 